\title{
High Dynamic Range Particle Image Velocimetry Applied to Heat Convection Studies
}

\author{
Tim Persoons ${ }^{1, \mathrm{a}}$ \\ ${ }^{1}$ Department of Mechanical \& Manufacturing Engineering, Parsons Building, Trinity College, Dublin 2, Ireland
}

\begin{abstract}
Convective heat transfer occurs in a wide range of engineering applications, from nuclear reactors to portable electronic devices. Accurate whole-field turbulence and flow measurements are crucial to understanding convective heat transfer in complex flow fields, thereby enabling optimal design of these devices. Particle image velocimetry (PIV) is the preferred whole-field flow measurement technique. However in many configurations the dynamic velocity range of conventional PIV is too limited to accurately resolve both high mean velocities and turbulence intensities in lower velocity regions. This paper employs high dynamic range (HDR) PIV with an advanced acquisition and processing technique based on multiple pulse separation (MPS) double-frame imaging. The methodology uses a conventional adaptive multi-grid algorithm for vector evaluation, and determines the optimal pulse separation in space and time in a post-processing routine. Two test cases are discussed: For an impinging synthetic jet flow (Case I), HDR PIV increases the dynamic velocity range 25 -fold compared to conventional PIV. For an oscillatory buoyant plume from a pair of horizontal heated cylinders (Case II), the dynamic velocity range is increased 5.5 times. This technique has yielded new insights in synthetic jet heat transfer by correlating local surface heat transfer rates to near-wall turbulence intensity in a single whole-field measurement.
\end{abstract}

\section{Introduction}

Section 1.1 reviews the key aspects of the dynamic velocity range of particle image velocimetry (PIV). Sections 1.2 and 1.3 review the existing literature related to the two test cases (I: impinging synthetic jet, II: buoyant plume from a horizontal heated cylinder pair).

\subsection{Dynamic velocity range of PIV}

The dynamic velocity range $D R_{V}$ is defined as the ratio of maximum to minimum resolvable velocity, or $D R_{V}=V_{\max } / \sigma_{V}=s_{\max } / \sigma_{s}$ where $\sigma_{V}$ and $\sigma_{s}$ are minimum resolvable velocity and particle displacement, respectively $\left(\sigma_{V}=M \sigma_{s} / \tau\right.$, where $M$ is the spatial pixel resolution and $\tau$ is the pulse separation time). The minimum resolvable displacement $\sigma_{s}$ is determined by the overall displacement uncertainty and bias error. As terminology, accuracy and error refer to systematic bias between measured and true values, whereas precision and uncertainty refer to the repeatability of the measurement.

As PIV evaluation methods have evolved over time, the dynamic velocity range has been steadily improved. For single-pass correlation algorithms, Keane and Adrian [1] proposed a quarter window rule $\left(s_{\max }<1 / 4 d_{I}\right)$ to avoid excessive loss of correlation strength, yielding $D R_{V}^{(S P)}=$

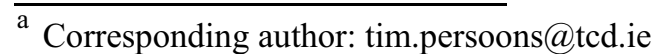

$\frac{1}{4} d_{I} / \sigma_{s}^{(S P)}$. The dependence of $\sigma_{s}^{(\mathrm{SP})}$ on a number of parameters (e.g., particle image displacement, density and diameter, interrogation window size, image noise, digitization and quantization, velocity gradients) has been reviewed extensively for single-pass correlation [2,3].

Using iterative window deformation with progressive grid refinement (multi-grid correlation), a tenfold reduction in uncertainty is achieved compared to singlepass correlation, with $\sigma_{s}^{(\mathrm{MG})} \cong 10^{-3}$ pixel based on an analysis with artificial particle images [4]. Multi-grid PIV partly decouples the maximum displacement and interrogation window size, since the quarter rule [1] only applies to the coarsest grid and not to subsequent passes on finer grids. With an initial interrogation window size $k_{g} d_{I}$ and a final size $d_{I}$ (where typically $k_{g}=2-4$ ), the dynamic range $D R_{V}^{(M P)}=\frac{1}{4} k_{g} d_{I} / \sigma_{S}^{(M P)}$.

However, most studies report uncertainty values based on simulation results using noiseless artificial images. In realistic laboratory conditions, image noise and velocity gradients typically yield much higher uncertainty values of around 0.1 pixel, in spite of using advanced evaluation algorithms [5]. A realistic dynamic velocity range for multi-grid $\operatorname{PIV}\left(k_{g}=4, d_{I}=16\right.$ pixel $)$ is about $D R_{V} \cong 160: 1$.

By locally increasing the pulse separation time, some studies have been able to increase the dynamic range 
significantly compared to conventional multi-grid PIV [68]. However, these multi-frame techniques are typically restricted to low speed flows, and since they are proposed as alternatives to multi-grid algorithms, they cannot benefit from advances in mainstream PIV methods. These shortcomings are avoided by the high dynamic range methodology of Persoons and O'Donovan [9] which is used in this paper.

\subsection{Case I: Forced convection heat transfer to an impinging synthetic jet}

An impinging synthetic jet can achieve high local heat transfer rates comparable to a continuous jet, yet without net mass inflow. Synthetic jets are zero net mass flux oscillatory flows, consisting of a train of propagating vortices formed by periodic ejection and suction of fluid across an orifice. Free synthetic jets have been studied extensively for applications in active flow control [10]. When impinging onto a heated surface, the periodic nature of the synthetic jet flow promotes entrainment, mixing and break-up of the thermal boundary layers.

An unconfined axisymmetric synthetic jet flow is characterised by two parameters: the dimensionless stroke length $L_{0} / D$ and the Reynolds number $R e=U_{0} D / v$, where $D$ is the orifice diameter, $L_{0}$ is the stroke length ( $\left.=\int_{t=0}^{1 /(2 f)} U_{m}(t) d t\right), U_{0}=f L_{0}$ and $U_{m}(t)$ is the oscillating mean orifice velocity. As such, $L_{0} / D$ is inversely proportional to a Strouhal number, $L_{0} / D=\left(f D / U_{0}\right)^{-1}$. An impinging jet is further characterised by the nozzle-tosurface spacing $H / D$. Studies of the flow field of free synthetic jets (e.g., Shuster and Smith [11]) have shown that $L_{0} / D$ and $R e$ are the appropriate flow scaling parameters, which has also been confirmed for an axisymmetric impinging synthetic jet [12].

Depending on the surrounding conditions and the level of confinement, a synthetic jet flow can be prone to recirculation which limits its heat transfer performance $[12,13]$. In practical cooling applications a forced crossflow is required to supply fresh coolant. However, Persoons et al. [15] have shown that the flow vectoring effect induced by adjacent free synthetic jets operating out of phase [16] remains present for impinging jets. The vectoring jets induce a cross-flow, thus eliminating the need for external flow forcing as well as increasing the overall heat transfer performance by nearly $100 \%$ compared to non-interacting jets [15].

For an impinging synthetic jet using a slot orifice, Gillespie et al. [17] determined that the highest average heat transfer is obtained for $0.8<H / L_{0}<3.2$. In the far field $\left(H>>L_{0}\right)$, the velocity has decayed too much. In the near field $\left(H<<L_{0}\right)$, preheated fluid is recirculated back into the jet cavity, decreasing the heat transfer performance. For a round impinging synthetic jet at $H / D$ $=9.5$, Pavlova and Amitay [18] show velocity and turbulence intensity distributions for $0.8<L_{0} / D<5.3$ and $140<\operatorname{Re}<740$, and conclude that the highest heat transfer rate occurs for $1.3<H / L_{0}<8.5\left(0.12<L_{0} / H<\right.$ $0.77)$.

Travnicek and Tesar [19] studied the mass transfer characteristics of an annular impinging synthetic jet. By varying the Strouhal number, the authors explicitly investigated the stroke length dependence of the flow field and mass transfer behaviour. Two different flow regimes are identified at low and high stroke length, showing a strong influence on the stagnation point mass transfer rate and the overall radial profile of the mass transfer coefficient.

Using particle image velocimetry (PIV) on a round impinging synthetic jet with $H=2 D$, Valiorgue et al. [12] have identified the ratio of stroke length to nozzle-tosurface spacing as a proper scaling parameter for an impinging synthetic jet. A critical stroke length of about $L_{0} / H \cong 2.5$ is observed: at low $L_{0} / H$ (i.e., $H>0.4 L_{0}$ ), the vortices develop and lose strength before impingement and the stagnation heat transfer rate increases with $L_{0}$. At high $L_{0} / H\left(H<0.4 L_{0}\right)$, the flow tends to an intermittent on/off flow resulting in a time-averaged recirculation vortex, and the stagnation heat transfer rate becomes nearly independent of $L_{0}$.

A more comprehensive study of the heat transfer to an axisymmetric impinging synthetic jet $[13,14]$ covers a range of $2<H / D<16,500<R e<1500$ and $2<L_{0} / D<$ 40. A general correlation for the stagnation Nusselt number has been established which is valid for the entire parameter range. Unlike other studies, it also accounts for the stroke length dependence. While Valiorgue et al. [12] identified two heat transfer and flow regimes for $H / D=2$, the more comprehensive study has identified four heat transfer regimes based on the ratio of stroke length to nozzle-to-surface spacing $L_{0} / H[13,14]$. Figure 1 shows the normalized stagnation Nusselt number as a function of $\left(L_{0}-L_{0}^{(f)}\right) / H$, where the formation threshold $L_{0}^{(f)}=$ $1.5 D$. The letters $\mathrm{A}, \mathrm{B}, \mathrm{C}, \mathrm{D}$ indicate the four heat transfer regimes identified $[13,14]$. In regime $\mathrm{A}, N u_{0}$ increases sharply with $L_{0}$ and reaches a local maximum. In regime $\mathrm{B}$ (which is not observed for $H / D=2$ [12]) $N u_{0}$ decreases to a local minimum and then gradually increases again with $L_{0}$ in regime $\mathrm{C}$, reaching a constant level in regime $\mathrm{D}$.

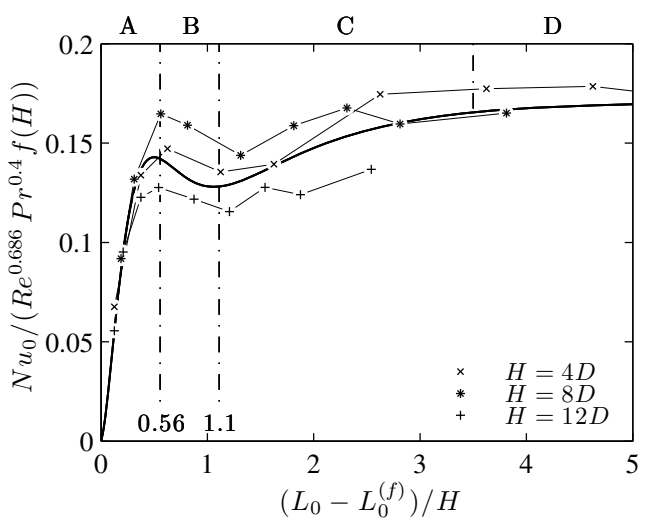

Figure 1. Dependence of normalised stagnation Nusselt number $N u_{0} /\left(\operatorname{Re}^{m} \operatorname{Pr}^{n} f(H)\right)$ on the ratio of stroke length to nozzle-tosurface spacing $\left(L_{0}-L_{0}{ }^{(f)}\right) / H$ (for $4 \leq H / D \leq 12,500 \leq R e \leq$ $1500,2<L_{0} / D<40$ ) indicating four heat transfer regimes A-D (see Persoons et al. $[13,14]$ )

A number of studies [12-20] have demonstrated the potential of synthetic jets for convective cooling. However, the understanding of the coupling between the 
fluid dynamics and the heat transfer mechanisms still falls short of that available for steady jets. Although the fluid dynamics of free synthetic jets have been studied extensively for flow control applications [10,11], the characteristics of impinging synthetic jets are less understood, and this requires accurate whole-field turbulence and flow measurements, using PIV. The ratio between the maximum velocity near the orifice to the low velocity in the outer regions and near the wall is typically about two orders of magnitude (100:1), which challenges even advanced PIV techniques [5].

\subsection{Case II: Natural convection heat transfer from a pair of horizontal heated cylinders}

Tubular heat exchangers are common devices for transferring heat between two fluid flows. The timeaveraged heat transfer characteristics are well known for forced convection from arrays of horizontal cylinders. However natural convection heat transfer has received less attention and there remain questions regarding the mechanisms, especially for closely packed tube arrays where thermal plumes of adjacent cylinders interact. Most studies have focused on averaged heat transfer characteristics for single cylinders [21] or arrays [22]. The interaction between cylinders is generally described as an overall heat transfer enhancement. Only limited knowledge exists on the influence of plume oscillations from one (upstream) cylinder on the heat transfer from another (downstream) cylinder, due to the difficulty in the transient numerical modelling of the plume.

Some papers discuss the swaying of a thermal plume from single cylinders in natural convection [23,24]. While low Rayleigh numbers are characterized by stationary two-dimensional laminar plumes, the plume starts to oscillate in irregular patterns at increasing $R a$. At high Rayleigh number $\left(R a>10^{10}\right)$, the plume transitions to the turbulent regime [24]. A greater body of research is available on swaying plumes from thin horizontal wires, regarded as line heat sources [25,26,27]. Desrayaud and Lauriat [28] investigated buoyancy induced flow from a horizontal wire heat source; for rectangular vessels two destabilizing mechanisms lead to low frequency motion due to instability of the buoyant plume. These mechanisms depend on the ratio of depth of immersion to vessel width. The oscillation frequency $f=0.0657 R a^{0.433}$ $\left(0.3 \cdot 10^{6}<R a<8 \cdot 10^{6}\right)$ [28] as per earlier results [27].

For a pair of cylinders, Eckert and Soehngen [29] found that the induced temperature and velocity fields due to the buoyant plume from downstream cylinders have opposing effects. The heat transfer from the upper cylinder decreases with decreasing cylinder spacing, due to a drop in local temperature difference. At larger spacing an increase in heat transfer occurs due to the higher local fluid velocity having a forced convection effect on the upper cylinder. Previous studies by the authors revealed a range of cylinder spacings and Rayleigh numbers showing beneficial interaction [30-32]. A spectral analysis of the surface heat flux indicated that during out-of-phase oscillations of adjacent plumes, beneficial mixing occurs which explains the heat transfer enhancement.

\subsection{Objectives}

Using high dynamic range (HDR) PIV based on multiple pulse separation (MPS) acquisition, this paper aims to obtain more accurate whole-field flow and turbulence measurements for use in experimental convective heat transfer studies. The technique will be demonstrated for two cases, an impinging synthetic jet and natural convection from horizontal heated cylinders.

\section{Experimental facilities}

\subsection{Impinging synthetic jet test facility (Case I)}

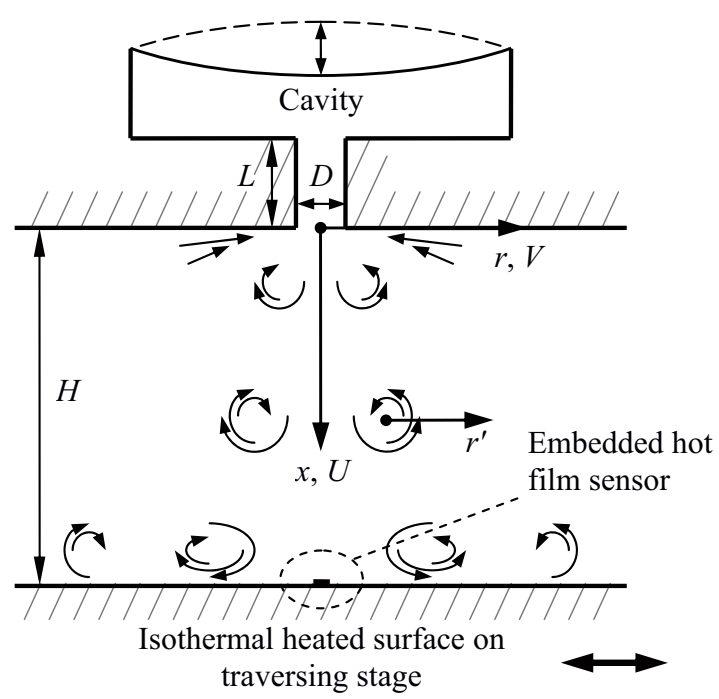

Figure 2. Schematic diagram of the impinging synthetic air jet facility $[12,13]$

Figure 2 shows a schematic diagram of the impinging synthetic air jet facility (orifice diameter $D=5 \mathrm{~mm}$, length $L=10 \mathrm{~mm}$ ), with the jet nozzle at a distance $H$ above a smooth planar impingement surface. The test facility is described in more detail elsewhere $[12,13]$.

The PIV system comprises a New Wave Solo-II $\mathrm{Nd}$ :YAG twin cavity laser $(30 \mathrm{~mJ}, 15 \mathrm{~Hz})$ and PCO Sensicam cooled CCD camera $\left(1280 \times 1024 \mathrm{px}^{2}, 12\right.$ bit $)$ with $28 \mathrm{~mm} \mathrm{f} / 2.8$ lens. The image magnification is about $1: 4(M=54 \mu \mathrm{m} / \mathrm{pixel})$. A glycol-water aerosol is used as seeding, with particle diameters between 0.2 and $0.3 \mu \mathrm{m}$. The particle image diameter is adjusted to about 2 pixel. Customised optics are used to generate a $0.3 \mathrm{~mm}$ thick light sheet in the $\{x, r\}$ plane through the orifice centre line (see Fig. 2). The camera is mounted perpendicular to the light sheet. A narrow band pass filter is used with fluorescent paint on the solid surfaces to maximise the signal-to-noise ratio near the walls.

Since this paper merely intends to demonstrate the benefits of high dynamic range PIV in a representative flow field, a single test condition is used $(H / D=8, R e=$ $\left.1500, L_{0} / D=3.4\right)$. As shown in Fig. 1 , a value of $\left(L_{0}-\right.$ $\left.L_{0}{ }^{(f)}\right) / H=0.24$ corresponds to regime 'A' [13]. The stagnation Nusselt number for these conditions is about $N u_{0} \cong 12$. A more comprehensive study of the interdependence of the flow field and heat transfer characteristics is given elsewhere $[12,13]$. 


\subsection{Natural convection test facility (Case II)}

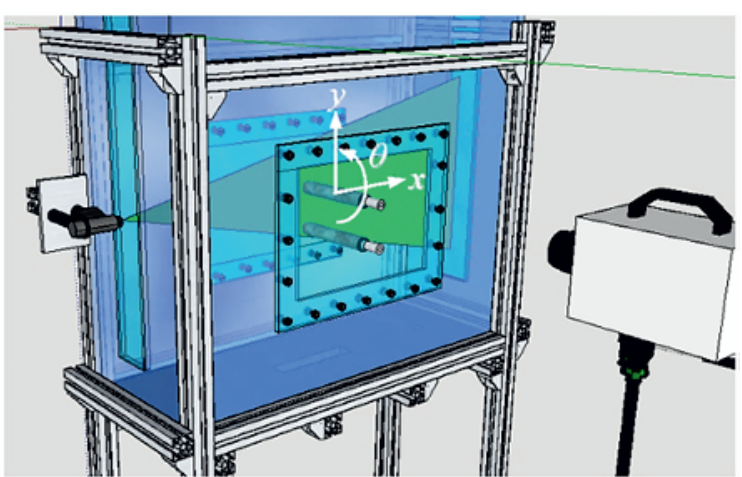

Figure 3. Diagram of the natural convection water tank test facility [32]

The natural convection test facility approximates the testing of a circular cylinder of infinite length surrounded by an infinite isothermal fluid. The facility uses a pair of isothermally heated copper cylinders with a diameter $D=$ $30 \mathrm{~mm}$. Confinement effects are minimized by an end plate spacing greater than $3 D$ and a depth of immersion $H$ greater than $3 D$ [32]. Figure 3 shows a schematic diagram of the facility built with these considerations in mind, measuring $900 \mathrm{~mm}$ high, $900 \mathrm{~mm}$ long and $300 \mathrm{~mm}(10 D)$ wide. Deoxygenated water was used as the working fluid. The large water volume (200 litres) minimises bulk water temperature drift during testing. The facility and procedures are described in more detail elsewhere [32].

Figure 3 depicts the arrangement of the light sheet intersecting the cylinders in the mid-plane of the vessel, and the double-frame camera (Photron Fastcam SA1, $1024^{2} \mathrm{px}, 5400 \mathrm{~Hz}$ at full frame rate, $12 \mathrm{bit}, 105 \mathrm{~mm} \mathrm{f} / 2.8$ lens). The light source is a Quantronix Darwin-Duo $\mathrm{Nd}$ :YLF double-pulsed laser $(15 \mathrm{~mJ}, 527 \mathrm{~nm})$, and the mean light sheet thickness is approximately $1 \mathrm{~mm}$. With the light sheet entering from the left, the cylinders cast a shadow where no PIV measurements are possible.

The camera is aligned nearly perpendicular to the light sheet, to minimize light reflections from the cylinder surface and maximize the visible region near the top of the cylinder. Because of the slightly off-perpendicular position (viewing angle of a few degrees), a calibration target was positioned in the water tank prior to the measurements to perform an optical distortion correction using Lavision Davis 7.2.2.

Polyamid particles $\left(30-70 \mu \mathrm{m}\right.$ in diameter, $\left.1.03 \mathrm{~g} / \mathrm{cm}^{3}\right)$ are used as seeding. The maximum Stokes number is below 0.2, indicating a negligible particle lag. After optical calibration of the camera setup in ambient conditions, the heating power is adjusted to the desired thermal conditions. As low frequency oscillations within the flow are expected, the camera frame rate is reduced to its minimum of $50 \mathrm{~Hz}$, providing a test duration of $26 \mathrm{~s}$. Local surface heat flux data is recorded simultaneously with the PIV images.

\section{Methodology: High dynamic range PIV}

Persoons and O'Donovan [9] proposed the multiple pulse separation (MPS) technique to achieve high dynamic range PIV results. The methodology is only briefly introduced here. Whereas multi-frame techniques $[7,8]$ acquire single-frame image sequences $\{\ldots, t, t+\delta t$, $t+2 \delta t, \ldots\}$, MPS PIV acquires double-frame images $\{\ldots$, $\left.\left[t, t+k_{\tau, 1} \tau_{\min }\right],\left[t+\delta t, t+\delta t+k_{\tau, 2} \tau_{\min }\right], \ldots\right\}$ with $N_{\tau}$ different pulse separation values $k_{\tau, i} \tau_{\min }\left(i=1 \ldots N_{\tau}\right)$ at a given frame rate $1 / \delta t$. Each image pair $\left[\mathrm{I}(0), \mathrm{I}\left(\tau_{i}\right)\right]$ with pulse separation $\tau_{i}=k_{\tau, i} \tau_{\min }$ is correlated using conventional multi-grid algorithms, resulting in displacement fields $\vec{s}_{i}=\vec{s}\left(x, y, \tau_{i}\right)$ (see Fig. 4).

By selecting a larger pulse separation $k_{\tau} \tau_{\text {min }}$ in the low velocity region (and a smaller pulse separation $\tau_{\min }$ in the high velocity region), the minimum measurable velocity $\sigma_{V} \propto \sigma_{s} /\left(k_{\tau} \tau_{\min }\right)$ and the dynamic range increases:

$$
D R_{V}^{(M P S)}=\frac{\frac{1}{4} k_{g} d_{I} / \tau_{\min }}{\sigma_{s}^{(M G)} /\left(k_{\tau} \tau_{\min }\right)}=k_{\tau} \cdot k_{g} \cdot \frac{\frac{1}{4} d_{I}}{\sigma_{s}^{(M G)}}
$$

The local multiplier $k_{\tau}$ is determined from the optimal pulse separation criterion. For each vector location, the optimal pulse separation corresponds to the maximum weighted peak ratio $Q^{\prime}$ which is a measure of local vector quality, combining correlation strength and precision:

$$
Q^{\prime}=Q\left(1-\frac{\sigma_{s}}{|s|}\right)
$$

where the peak ratio $Q$ measures correlation strength [1], and $\left(1-\sigma_{s} /|s|\right)$ estimates the local precision by comparing the displacement magnitude $|s|$ to the estimated minimum resolvable displacement $\sigma_{s}$. Typical values of $0.02<\sigma_{s}<$ 0.1 pixel are assumed, constant within the field of view [9]. Here, $\sigma_{s}=0.05$ pixel is taken. Further improvements to this simple approach can be made by locally estimating the uncertainty [33].

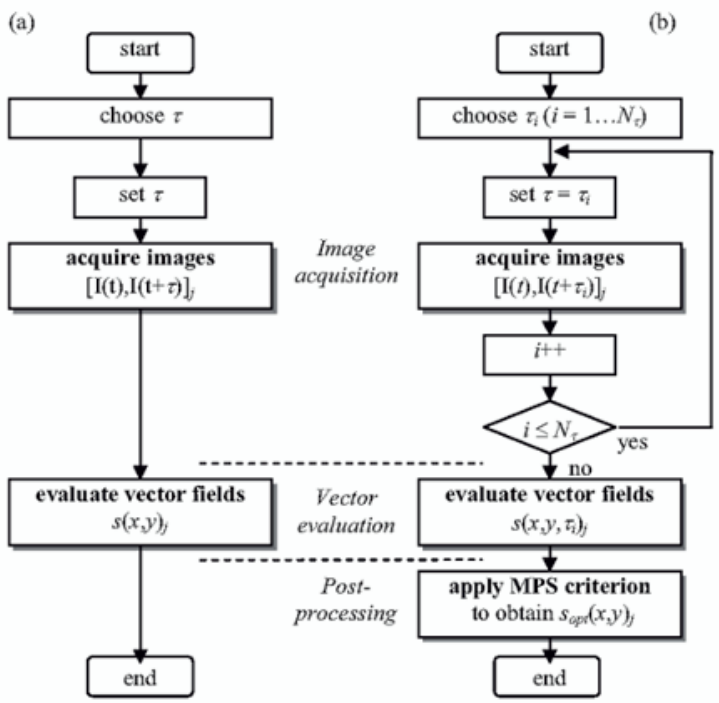

Figure 4. Flowchart for (a) conventional double-frame PIV and (b) high dynamic range (HDR) PIV using the multiple pulse separation (MPS) method [9]

The HDR velocity fields are reconstructed as $\vec{U}^{(H D R)}(x, y, t)=\operatorname{sel}_{Q^{\prime}}\left(\vec{U}_{i}\left(x, y, t, \tau_{i}\right)\right)$ based on a relaxed maximum operator with local $Q^{\prime}$ weighting:

$$
\operatorname{sel}_{Q^{\prime}}\left(a_{i}\right)=\frac{\sum a_{i} w_{i}}{\sum w_{i}} \text { with } w_{i}=\left[\frac{Q_{i}^{\prime}-\min _{i}\left(Q_{i}^{\prime}\right)}{\max _{i}\left(Q_{i}^{\prime}\right)-\min _{i}\left(Q_{i}^{\prime}\right)}\right]^{p}
$$


where the exponent $p>1$. As $p \rightarrow \infty$, the relaxed maximum reverts to a strict maximum selector. A finite exponent $p \cong 5$ is used here.

The choice of the $N_{\tau}$ pulse separation multipliers $k_{\tau, i}$ is arbitrary. The smallest pulse separation $\tau_{\min }$ limits the loss of correlation in the high velocity region, while the greatest multiplier $k_{\tau, N}$ can be determined similarly for the low velocity region, e.g. $k_{\tau, N}=U_{\max } / U_{\min }$. The increase in dynamic range $D R_{V}^{(M P S)} / D R_{V}^{(M G)}=k_{\tau, \text { eff }}$, where the effective multiplier $k_{\tau, \text { eff }} \leq \max \left(k_{\tau, i}\right)$ is the $95 \%$ percentile value of the $\tau_{\text {opt }} / \tau_{\min }$ histogram.

\section{Experimental results and discussion}

\subsection{Case I: Impinging synthetic jet flow}

This section discusses the turbulence field and surface heat transfer characteristics for an impinging round synthetic jet at $R e=1500, L_{0} / D=3.4$ and $H / D=8$. As shown in Fig. 1, the heat transfer strongly depends on stroke length since $\left(L_{0}-L_{0}^{(f)}\right) / H=0.24$ [13]. The formed vortices are just strong enough to propagate to the heat transfer surface, yet upon impingement rapidly break up into turbulence $[13,14]$. Therefore, the stagnation and wall jet regions are characterised by low level periodic velocity fluctuations and unresolved turbulence. These fluctuations are small compared to the velocity scale $U_{0}$ driving the vortex formation, yet nevertheless determine the surface heat transfer rate. The wide velocity range makes this an ideal case to demonstrate the benefits of HDR PIV.

In the following, the HDR results are obtained from seven pulse separation values $\left(\tau_{\min }=20 \mu \mathrm{s}\right.$, and $\tau / \tau_{\min }=$ $\{1,2,4,10,20,40,100\})$, with $\sigma_{s}=0.05 \mathrm{px}$ and $p=5$.

\subsubsection{Time-averaged flow and turbulence field}

Figure 5 shows the time-averaged velocity vectors in one half plane of the axisymmetric impinging jet flow. For clarity, only a selected number of vectors are shown. The background shading and isocontour lines represent the turbulent fluctuation magnitude $\left(u_{t}^{\prime 2}+v_{t}^{\prime 2}\right)^{1 / 2} / U_{0}$, with contour levels at $0.025,0.05,0.1,0.25,0.5$ and 1 . The conventional PIV results (Fig. 5a,b) show noisy vectors and overestimate turbulence intensity in the entrainment region (top right) because of the low velocities there $(\cong$ $\left.0.01 U_{\max }\right)$. Increasing the pulse separation time from $20 \mu \mathrm{s}$ (Fig. 5a) to $40 \mu$ s (Fig. 5b) slightly improves the results in the entrainment region, which however reduces the accuracy near the orifice. In Fig. 5a,b the jet core region and the shear layer is well captured, yet unphysical behaviour is shown (i) close to the upper wall and (ii) in the outer wall jet. This is due to insufficient dynamic range and the small near-wall mean velocity. By contrast, the HDR PIV results in Fig. 5c show fewer noisy vectors in the bulk flow, and near-wall velocity vectors which are correctly tangent to the walls.

Compared to the conventional PIV approach with $\tau=$ $20 \mu \mathrm{s}$, the HDR PIV approach features a 25 -fold or $28 \mathrm{~dB}$ increase in dynamic velocity range.
Assuming the HDR results are the reference (as did Stanislas et al. [5]), the maximum contribution to the velocity uncertainty due to the limited dynamic range of conventional PIV is about $100 \% U_{0}$ (for $20 \mu \mathrm{s}$ ) and $50 \% U_{0}$ (for $40 \mu \mathrm{s}$ ). These values are based on the difference in turbulence intensity observed between Figs. 5a,b and Fig. $5 c$. While this worst case scenario applies only to two small regions, the majority of the field (excluding the jet core) shows increased uncertainty levels of $10 \% U_{0}$ (for $20 \mu \mathrm{s}$ ) and $5 \% U_{0}$ (for $40 \mu \mathrm{s}$ ).

Figure 6 shows cross-stream profiles of the (a) axial and (b) radial turbulence intensity $u^{\prime} / U_{0}$ and $v^{\prime} / U_{0}$ at four distances from the orifice. The HDR results are represented by the solid lines with circular markers $(-\bigcirc-)$. The other markers represent conventional PIV results at $(\diamond) 20 \mu \mathrm{s}$ and $(\square) 40 \mu \mathrm{s}$. For a free synthetic jet at $L_{0} / D=3$ and $R e=2500$, Shuster and Smith [11] show similar turbulence intensity profiles, which are in excellent agreement with the HDR results in Fig. $6(-\circ-)$. The axial turbulence intensity $u^{\prime} / U_{0}$ features a strong peak along the jet centreline, whereas the radial turbulence intensity $v^{\prime} / U_{0}$ shows a distinct double peaked behaviour up to $x / D<7$, due to the periodic passage of propagating vortices. Beyond $x / D>7$, the vortices in the free synthetic jet begin to lose phase coherence and the turbulence profiles rapidly spread out radially. Due to the presence of the impingement surface at $H / D=8$, the comparison with data from Shuster and Smith [11] only holds in the free jet region $(x / D<7)$.

The conventional PIV results in Fig. $6(\diamond, \square)$ show some inconsistencies. While the results for $20 \mu \mathrm{s}(\diamond)$ are accurate in the jet core, they overestimate the turbulence intensity in the outer shear layer by $10-20 \% U_{0}$, and beyond $r / D>4$ by up to $60 \% U_{0}$. The results for $40 \mu \mathrm{s}(\square)$ are more accurate in the low velocity region yet underestimate the axial velocity fluctuation intensity close to the orifice (by $50 \% U_{0}$ ) while overestimating the radial component at the same location (by $40 \% U_{0}$ ). This is due to strong velocity gradients causing a higher incidence of spurious vectors at this greater pulse separation time value.

Other flow field characteristics measured with MPS PIV such as the jet half-width and the vortex evolution are also in excellent agreement with results by Shuster and Smith [11].

\subsubsection{Time-averaged radial profiles of Nusselt number and near-wall turbulence intensity}

The lines in Fig. 7 represent the cyclic (phaseresolved) fluctuation intensity at $0.5 \mathrm{~mm}(=0.1 D)$ above the impingement surface, determined using conventional PIV with $\tau=20 \mu$ s (Fig. 7a), $\tau=40 \mu$ s (Fig. 7b), while Fig. $7 \mathrm{c}$ is obtained using HDR PIV. Each plot also shows the radial profile of the local time-averaged Nusselt number (normalised with the time-averaged stagnation point value $N u_{0}$ ) as hollow markers. The fluctuating local Nusselt number $N u^{\prime}(\triangle)$ is defined as the standard deviation of the instantaneous local Nusselt number, or $N u^{\prime}(r)=\left[N_{i}^{-1} N_{\theta}^{-1} \sum_{i} \sum_{\theta}\left(N u_{i}(r, \theta)-N \bar{u}(r)\right)^{2}\right]^{1 / 2}$ where $N \bar{u}(r)$ $=N_{i}^{-1} N_{\theta}^{-1} \sum_{i} \sum_{\theta} N u_{i}(r, \theta)$. 

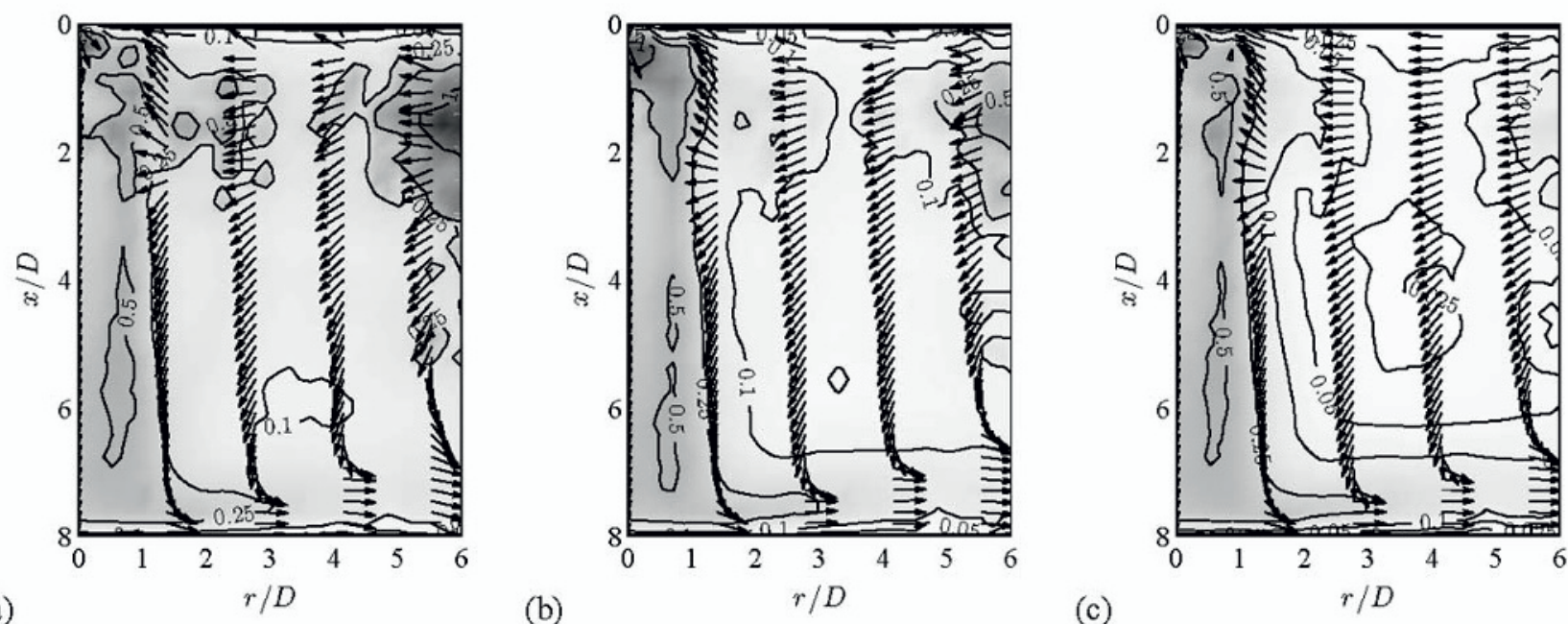

Figure 5. Impinging synthetic jet: Time-averaged normalised velocity vectors with contours of unresolved turbulent fluctuation magnitude $\left(u_{t}^{\prime 2}+v_{t}^{\prime 2}\right)^{1 / 2} / U_{0}$, using (a) conventional PIV with $\tau=20 \mu \mathrm{s}$, (b) $\tau=40 \mu$ s and (c) HDR PIV with $N_{\tau}=7$ pulse separation values $20 \mu \mathrm{s} \leq \tau \leq 2000 \mu \mathrm{s}\left(\sigma_{s}=0.05 \mathrm{px}, p=5\right)$
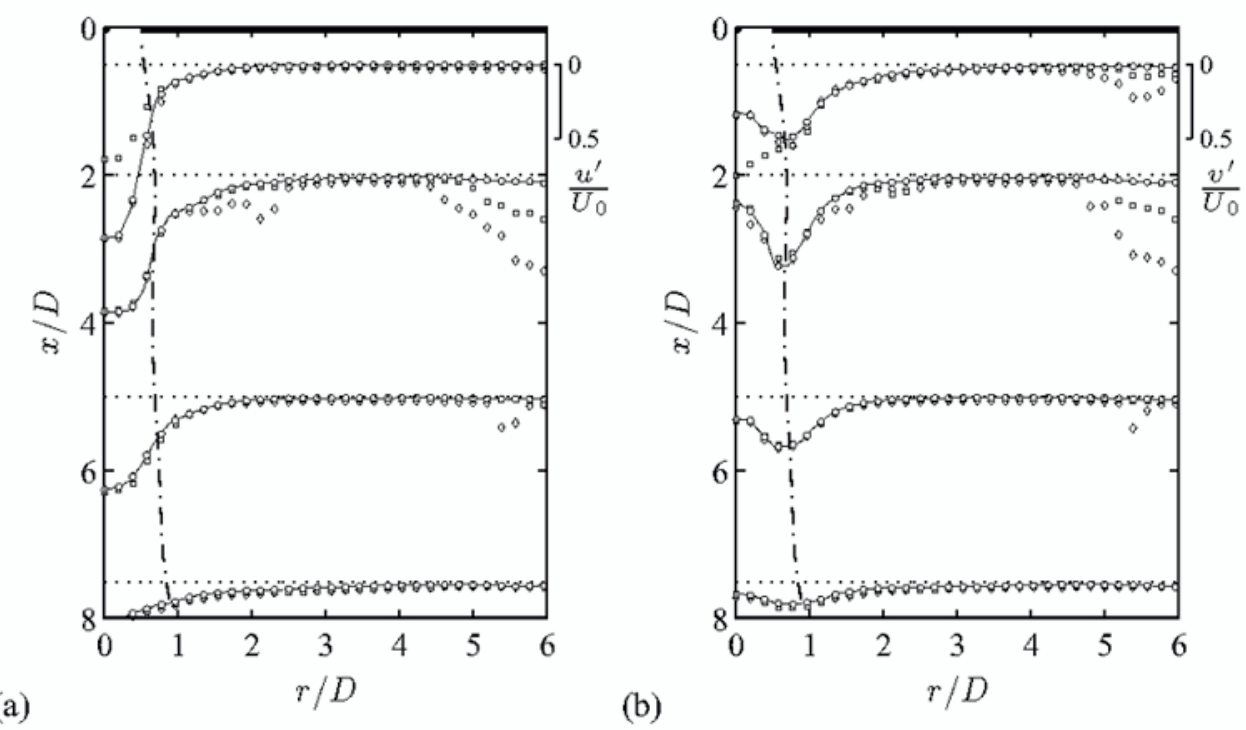

Figure 6. Time-averaged radial profiles (at $x / D=0.5,2,5$ and 7) of (a) axial and (b) radial velocity fluctuation intensity $u^{\prime} / U_{0}$ and $\nu^{\prime} / U_{0}$ using $(\diamond)$ conventional PIV with $\tau=20 \mu \mathrm{s}$ and $(\square) \tau=40 \mu \mathrm{s}$, compared to (-○-) HDR PIV $\left(N_{\tau}=7,20 \mu \mathrm{s} \leq \tau \leq 2000 \mu \mathrm{s}, \sigma_{s}=\right.$ $0.05 \mathrm{px}, p=5)$

(a)

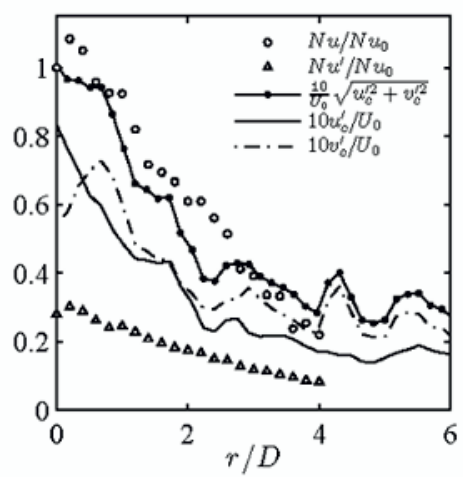

(b)

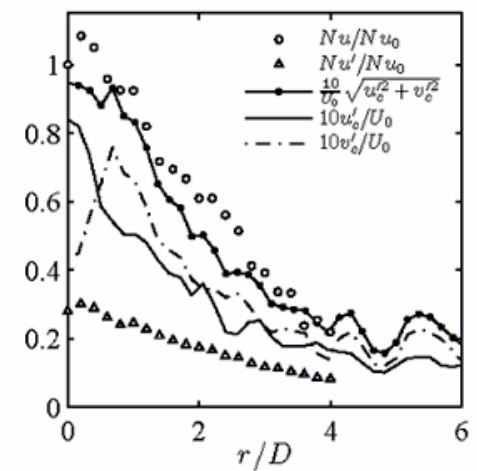

(c)

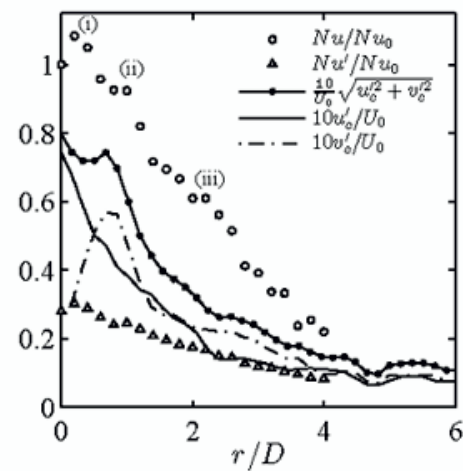

Figure 7. Radial profiles of time-averaged and fluctuating Nusselt number $\left(O N u / N u_{0}, \triangle N u^{\prime} / N u_{0}\right)$ and the cyclic fluctuation intensity $u_{c}^{\prime} / U_{0}$ and $v_{c}{ }^{\prime} / U_{0}$ at $0.5 \mathrm{~mm}$ above the surface: (a) Conventional PIV at $\tau=20 \mu \mathrm{s}$ and (b) $\tau=40 \mu \mathrm{s}$, (c) HDR PIV $\left(N_{\tau}=7,20 \mu \mathrm{s} \leq \tau \leq\right.$ $2000 \mu \mathrm{s}, \sigma_{s}=0.05 \mathrm{px}, p=5$ ). Peaks in the time-averaged Nusselt number are indicated at (i) $r / D=0.2$, (ii) $r / D=0.8$, (iii) $r / D=2$ 
The time-averaged Nusselt number exhibits a local reduction at the stagnation point, a minor peak at $r / D=$ 0.2 (indicated as (i) in Fig. 7c), a minor secondary peak at (ii) $r / D \cong 0.8$ and a minor third peak at (iii) $r / D \cong 2$. The profile of $N u^{\prime}(r)$ is nearly proportional to $N u(r)$ in the stagnation zone $(r / D<2)$, although the ratio $N u^{\prime}(r) / N u(r)$ increases as $r / D>2$.

The local Nusselt number is expected to depend to some extent on the local turbulence intensity, determined by the wall-normal $\left(u_{c}^{\prime} / U_{0}\right)$ and tangential $\left(v_{c}^{\prime} / U_{0}\right)$ components. For a simple case such as the stagnation point of a steady impinging jet, this dependence could take the form of a linear scaling $\left(N u \propto\left(u_{c}{ }^{2}+v_{c}{ }^{2}\right)^{1 / 2}\right)$ $[34,35]$. In the wall jet region of a steady impinging jet, the scaling between Nusselt number and turbulence quantities is more complicated, yet typically locations of heat transfer peaks and maximum turbulence intensity can still be matched [36].

Using conventional PIV (Fig. 7a,b), the near-wall turbulence profiles show a reasonable agreement with the local Nusselt number, however in the outer region $(r / D>$ 4) the turbulence measurement becomes unreliable due to excessive noise in the velocity vectors as a result of insufficient dynamic velocity range. The limited accuracy of the turbulence profiles in Fig. 7a,b prevents a detailed comparison with the heat transfer profile.

The higher dynamic velocity range of MPS PIV yields more accurate vectors in regions of low timeaveraged velocity, thereby avoiding a systematic overestimation of the turbulence. For a steady jet, similar improvements have been shown [9].

Using MPS PIV, the near-wall turbulence profiles in Fig. $7 \mathrm{c}$ are much smoother and more reliably match corresponding features in the Nusselt profiles. Figure $7 \mathrm{c}$ shows that the radial velocity fluctuation $v_{c}{ }^{\prime}$ increases from a minimum near the stagnation point to a pronounced peak at $r / D=0.8$, which coincides with the small secondary peak in the $N u$ and $N u^{\prime}$ profiles at the same location. Beyond the stagnation zone, $v_{c}{ }^{\prime}$ rapidly drops in the region $0.8<r / D<1.5$, followed by a more gradual decrease for $r / D>1.5$.

The wall-normal velocity fluctuation $u_{c}{ }^{\prime}$ exhibits a sharp peak at the stagnation point $(r / D=0)$, followed by a monotonic decrease. Although the overall fluctuation magnitude does not show a reduction at the stagnation point, the small transverse fluctuation $v_{c}{ }^{\prime}$ may be contributing to the minor reduction in stagnation point Nusselt number. Beyond the stagnation zone, $u_{c}{ }^{\prime}$ experiences a small but sudden decrease at $r / D=2$ which is reflected in the reduction of $N u$ beyond the third peak at the same location.

Based on this comparison, it seems that the coupling between heat transfer and near-wall turbulence in an impinging synthetic jet is more complicated than in a steady jet [34-36]. This is not entirely unexpected since a synthetic jet flow features both stronger velocity and temperature fluctuations because of the enhanced mixing and entrainment compared to steady jets. McGuinn et al. [37] showed a strong coherence between fluid temperature fluctuations and Nusselt number, especially at low Reynolds number. Near-wall velocity fluctuations and fluid temperature both contribute to the resulting heat transfer rate profile. This becomes even clearer in the following sections, comparing the phase-resolved heat transfer and velocity data.

Figure 8 compares conventional PIV and MPS PIV results in terms of the phase-resolved evolution of the near-wall stagnation point velocity and turbulence intensity. The horizontal scale is centred around the vortex impingement at $\theta-\theta_{\max } \cong 720^{\circ}$. For clarity, the phase-resolved Nusselt number $N u(r, \theta)(\bigcirc)$ is plotted after subtracting the local time-averaged component $N \bar{u}(r)$ and normalising with the stagnation Nusselt number $N u_{0}$. The triangular markers $(\triangle)$ represent the unresolved fluctuating Nusselt number $N u_{t}^{\prime} / N u_{0}$, defined similarly to the unresolved turbulence velocity fluctuation as the root mean square deviation around the phaseresolved Nusselt number, or $N u_{t}^{\prime}(r, \theta)=\left[\begin{array}{ll}N_{i}^{-1} & \sum_{i}\end{array}\right.$ $\left.\left(N u_{i}(x, r, \theta)-N u(r, \theta)\right)^{2}\right]^{1 / 2}$ where $N u(r, \theta)=N_{i}^{-1} \sum_{i}$ $\left(N u_{i}(x, r, \theta)\right.$.

Figure $8 \mathrm{c}$ shows a qualitative agreement between the local heat transfer coefficient and the phase-resolved HDR velocity and turbulence intensity. The vortex impingement exhibits a sudden increase in turbulence intensity as the outer shear layer reaches the surface $(\theta-$ $\theta_{\max }=630^{\circ}$ ). The turbulence intensity remains quite constant during the impingement process. The phaseresolved Nusselt number increases more gradually and slightly lags the near-wall velocity and turbulence evolution. From $\theta-\theta_{\text {max }} \cong 765^{\circ}$ until $810^{\circ}$, the turbulence intensity decreases to the level before impingement, which is accompanied by a more gradual reduction in Nusselt number. The unresolved Nusselt number fluctuation amplitude is fairly constant in time, with a slight fluctuation in line with the phase-resolved Nusselt number which peaks at the end of the impingement around $\theta-\theta_{\max } \cong 765^{\circ}$. The phase lag between the Nusselt number and the flow quantities indicates a mixed effect of temperature and velocity fluctuations [37].

In summary for Case I, Figs. 5-8 demonstrate the benefits of the improved dynamic velocity range of MPS PIV. Using the HDR approach, phase-resolved turbulence intensity and velocity data are less contaminated by poorly resolved vectors in regions of low time-averaged velocity. This allows more reliably identifying the physical trends and establishing the governing heat transfer mechanisms in complicated convection flows.

\subsection{Case II: Natural convection from adjacent horizontal cylinders}

As described in a recent study by Persoons et al. [32], the natural convection flow field around adjacent horizontal heated circular cylinders is characterized by strong velocity gradients and differences in velocity magnitude between the thermal plume and the entrainment region. As such, the same high dynamic range PIV technique using multiple pulse separation (MPS) acquisition and processing is applied. The HDR results are obtained using three pulse separation values $\left(\tau_{\min }=40 \mathrm{~ms}\right.$, and $\left.\tau / \tau_{\min }=\{1,2,4\}\right)$, with $\sigma_{s}=0.05 \mathrm{px}$ and $p=5$. 
(a)

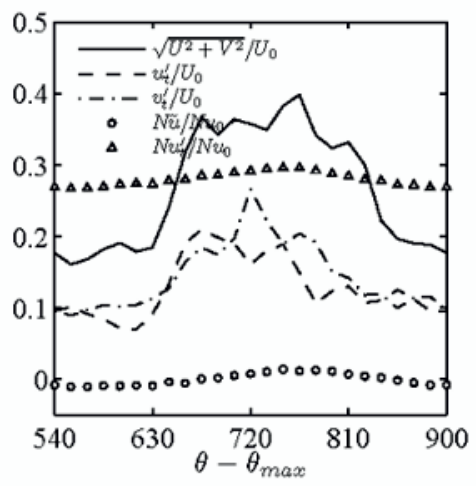

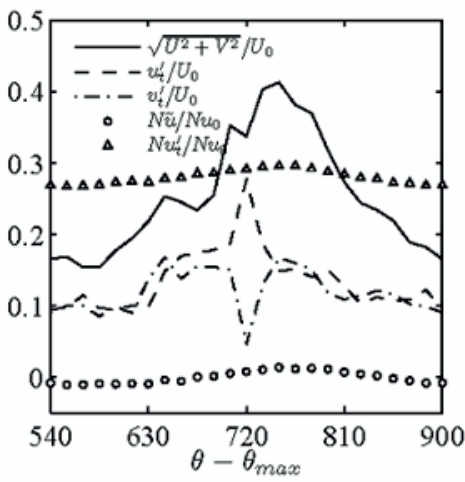

(c)

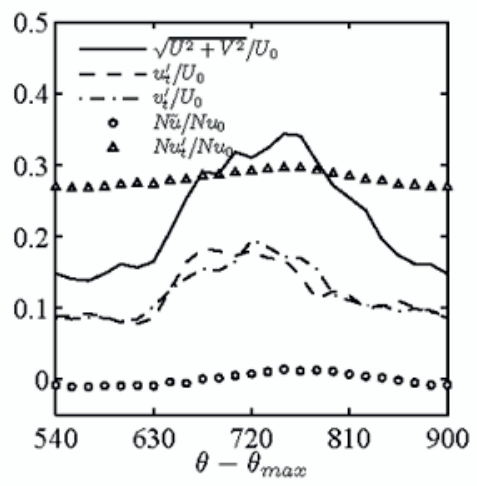

Figure 8. Phase-resolved Nusselt number $N \tilde{u}(r, t) / N u_{0}=(N u(r, t)-N \bar{u}(r)) / N u_{0}(O)$ and unresolved fluctuating Nusselt number $N u_{t}^{\prime} / N u_{0}$ $(\triangle)$, velocity (-) and unresolved turbulence intensity $u_{t}^{\prime} / U_{0}(--), v_{t}^{\prime} / U_{0}(-\cdot-)$ at $0.5 \mathrm{~mm}$ above the surface and $r=0$. (a) Conventional PIV at $\tau=20 \mu \mathrm{s}$ and (b) $\tau=40 \mu \mathrm{s}$, (c) $\operatorname{HDR} \operatorname{PIV~}\left(N_{\tau}=7,20 \mu \mathrm{s} \leq \tau \leq 2000 \mu \mathrm{s}, \sigma_{s}=0.05 \mathrm{px}, p=5\right)$
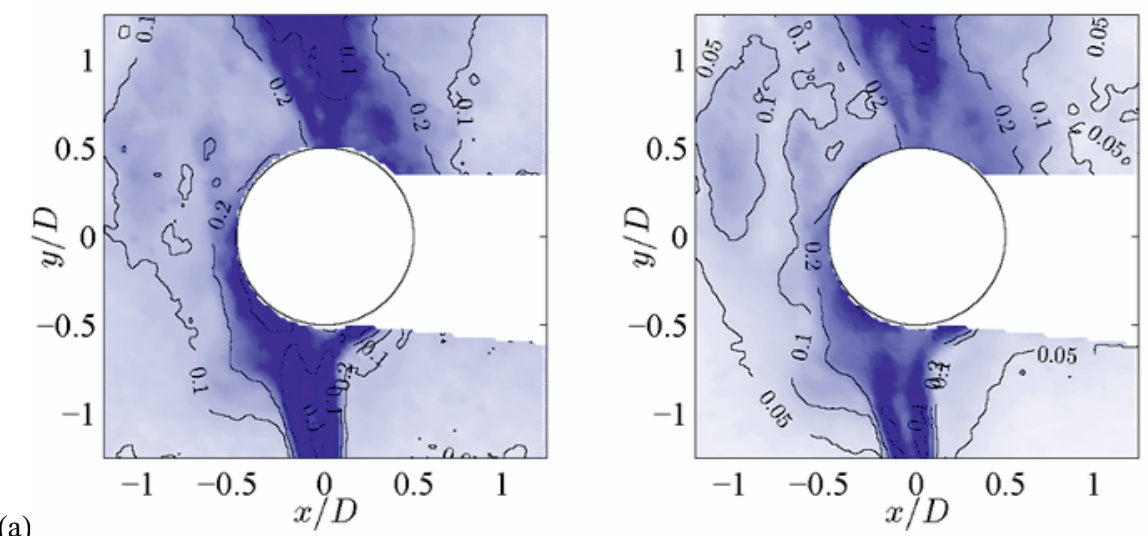

Figure 9. Natural convection from heated cylinder pair: Instantaneous turbulence intensity distribution $\left(u^{2}+v^{2}\right)^{1 / 2} / V_{\text {ref }}$ around the upper cylinder at $R a=3.6 \times 10^{6}$ and $S=3 D$, using (a) conventional PIV with $\tau=40 \mathrm{~ms}$ and (b) HDR PIV with $N_{\tau}=3$ pulse separation values $\tau=40,80$ and $160 \mathrm{~ms}\left(\sigma_{s}=0.05 \mathrm{px}, p=5\right)$
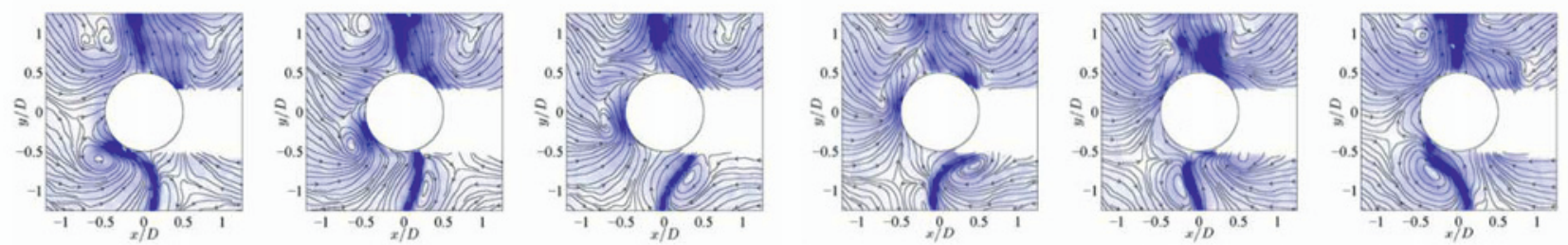

Figure 10. Instantaneous streamline and velocity magnitude plots at $t=0 \mathrm{~s}$ to $12.5 \mathrm{~s}$ in steps of $2.5 \mathrm{~s}$ (left to right) showing one plume oscillation period around the lower stagnation point of the upper cylinder at $R a=3.7 \times 10^{6}$ and $S=3 D\left(V_{\text {ref }}=4.2 \mathrm{~mm} / \mathrm{s}\right.$, plume width $2 b$ $=0.42 D$ at $y=-1 D)$

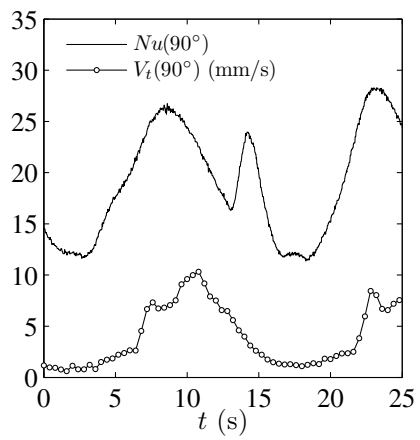

Figure 11. Simultaneously acquired time-resolved $(-)$ local $N u$ on left side of upper cylinder $\left(\theta=90^{\circ}\right)$ and $(-O-)$ near-cylinder tangential velocity $V_{t}$ (in $\mathrm{mm} / \mathrm{s}$ ) just above the sensor position, at same conditions as Fig. 10 
Figure 9 shows an arbitrary instantaneous turbulence intensity field using (a) conventional PIV and (b) HDR PIV. The reference plume velocity is defined as

$$
V_{\text {ref }}=\frac{1}{b} \int_{x} \bar{V}(x, y) d x
$$

where the plume width $b$ is defined as the distance between the locations where the upward velocity drops to $25 \%$ of the peak velocity. Although the plume width slightly increases with $y$, the value of $V_{\text {ref }}$ according to Eq. (4) is reasonably independent of $y$. Within the Rayleigh number range investigated the plume width $b$ remained constant at $0.35 \mathrm{D}(=10.5 \mathrm{~mm}$; evaluated at $y=$ $2 D$ ). The plume velocity according to Eq. (4) is typically about $1 / 4$ of the characteristic velocity based on the square root of the Grashof number, $(\mathrm{Ra} / \mathrm{Pr})^{1 / 2} v / \mathrm{D}$.

Figure 9a shows that conventional PIV tends to overestimate turbulence levels in near stagnant regions, as shown for Case I. Table 1 compares the dynamic measurement range for Figs. $9 a$ and $9 b$. The MPS technique increases the dynamic range for mean velocity and turbulence intensity 5.5-fold compared to conventional PIV. While the enhancement is smaller than the 25-fold increase seen in Case I, the measurement accuracy nevertheless benefits from the HDR approach.

Table 1. Comparison of the dynamic measurement range of the mean velocity and turbulence intensity using conventional and HDR PIV, for $R a=3.6 \times 10^{6}$ and $S=3 D$ (see Fig. 9)

\begin{tabular}{ccc}
\hline Quantity & \multicolumn{2}{c}{ Measurable range } \\
& Conventional PIV & HDR PIV \\
\hline time-averaged & 0.028 to $2.06 \mathrm{~mm} / \mathrm{s}$ & 0.005 to $1.86 \mathrm{~mm} / \mathrm{s}$ \\
velocity $\left(U^{2}+V^{2}\right)^{1 / 2}$ & $\mathrm{DR}_{U}=70: 1$ & $\mathrm{DR}_{U}=410: 1$ \\
\hline rms turbulence & 0.063 to $1.38 \mathrm{~mm} / \mathrm{s}$ & 0.012 to $1.36 \mathrm{~mm} / \mathrm{s}$ \\
intensity & $\mathrm{DR}_{u^{\prime}}=20: 1$ & $\mathrm{DR}_{u^{\prime}}=110: 1$ \\
$\left(u^{, 2}+v^{, 2}\right)^{1 / 2}$ & & \\
\hline
\end{tabular}

Figure 10 shows a single period of a buoyant plume oscillation in the region between a pair of vertically aligned cylinders at $R a=3.7 \times 10^{6}$ and $S=3 D$. From left to right, the plots represent instantaneous flow fields at 2.5 second intervals. The buoyant plume from the lower cylinder does not split and rise symmetrically around the upper cylinder, but instead oscillates back and forth across the upper cylinder. As a result of the swaying motion, the cylinder experiences alternating pockets of high velocity fluid passing along its sides. Studies by Sadeghipour and Asheghi [38] and Sparrow and Niethammer [39] attributed heat transfer enhancement from the upper cylinder to the forced convection effect due the lower cylinder's plume. However these flow field measurements suggest that lateral plume oscillation may represent an additional enhancement mechanism.

Figure 11 shows the local Nusselt number time trace at a circumferential position of $90^{\circ}$ (left side) on the upper cylinder. The circular markers represent the simultaneously measured tangential velocity $V_{t}$ near the heat flux sensor. $V_{t}$ is determined from the time-resolved PIV data, by averaging the tangential velocity in the boundary layer up to $0.2 \mathrm{D}$ from the cylinder surface:

$$
V_{t}\left(\theta=90^{\circ}, t\right)=\frac{1}{0.2 D} \int_{r=0.5 D}^{0.7 D} V_{t}\left(r, \theta=90^{\circ}, t\right) d r
$$

Figure 11 demonstrates that the time-resolved Nusselt number $N u\left(90^{\circ}\right)$ and the nearby tangential velocity $V_{t}$ correlate well. The spectrum of both signals exhibits a peak fluctuation frequency at $0.065 \mathrm{~Hz}( \pm 0.003 \mathrm{~Hz})$, with only a minor phase lag between the local velocity and Nusselt number. Figure 11 also reveals some higher order components in both heat transfer and plume velocities. As shown in Fig. 10, the flow field indeed features a wide range of vortices down to small eddies. Stochastic phenomena such as vortex shedding, merging and dissipation into small-scale turbulence can explain these higher order perturbations observed in Fig. 11.

A possible interpretation for the plume swaying is that the vortices formed near the bottom of the upper cylinder are governed by a mechanism similar to KelvinHelmholtz instability in wake flows. The shear layers in the wake of a single cylinder in cross-flow are known to organise into a Karman vortex street. Expressed as Strouhal number, the dimensionless shedding frequency is quite constant over a wide range of Reynolds numbers based on approach velocity and cylinder diameter $(300<$ $R e<10^{5}$ ) [40]. For Fig. 11 (at $R a=3.7 \times 10^{6}$ and $S=3 D$ ), the fluctuation frequency $f=0.065 \mathrm{~Hz}$ becomes

$$
S r=\frac{f D}{V_{\text {ref }}} \cong 0.46( \pm 0.02)
$$

where $V_{\text {ref }}$ is determined according to Eq. (4) as the average plume velocity at a distance of $0.5 D$ below the upper cylinder. Using the peak centreline plume velocity instead as reference velocity in Eq. (6) yields $S r=0.30( \pm$ $0.01)$. Although clearly pressure-driven flow across a single cylinder differs from buoyancy-driven flow across a pair of cylinders, the peak Strouhal number is of the same magnitude as the natural shedding frequency for a cylinder in cross-flow, $S r=0.19( \pm 0.01)$ [40].

\section{Conclusions}

The paper has demonstrated the benefits of high dynamic range (HDR) particle image velocimetry for two heat convection test cases, an impinging synthetic jet flow and natural convection from a pair of heated horizontal cylinders. The HDR PIV approach [9] uses multiple pulse separation (MPS) acquisition to increase the dynamic velocity range compared to conventional double-frame multi-grid PIV. The MPS technique is applied in a post-processing step, and poses no restrictions to using advanced vector evaluation methods such as multi-grid correlation with window deformation.

For Case I (impinging synthetic jet flow), the dynamic velocity range using HDR PIV has increased 25-fold compared to the best possible conventional PIV measurement. Based on a minimum resolvable particle displacement of 0.1 pixel in realistic conditions [5], the dynamic velocity range thus increases from about 160:1 $(44 \mathrm{~dB})$ to $4000: 1(72 \mathrm{~dB})$. As a result, the noise level in the turbulence measurements is significantly reduced. The improvement is most pronounced in low mean velocity regions (e.g., the outer wall jet and entrainment region), and also in regions with strong temporal velocity fluctuations, near the orifice and the stagnation zone. 
For Case II (natural convection from a pair of heated cylinders), MPS PIV has increased the dynamic range in measuring the velocity and turbulence fields. In typical conditions, a 5.5-fold increase in dynamic velocity range has been obtained, resulting in more accurate flow and turbulence fields. Compared to Case I, the benefit was less pronounced in this flow of moderate dynamic range.

For Case I however, some new insights into the heat transfer mechanisms in an impinging synthetic jet were obtained because of the enhanced high dynamic range:

- The phase-resolved profiles of near-wall turbulence and Nusselt number shows an evolving high turbulence region formed by the impinging vortex. This zone moves radially outwards and dissipates in the wall jet region, at $r / D=2$. This confirms the identified heat transfer and flow regimes $[13,14]$.

- The phase-resolved Nusselt number $N u(r, \theta)$ slightly lag the velocity and turbulence intensity in the inner stagnation zone $(r / D<0.5)$, indicating a mixed effect of fluid temperature and velocity fluctuations. The Nusselt number loses coherence at the edge of the stagnation zone $(r / D=0.8)$ although the flow still exhibits a pronounced periodicity.

- Compared to a steady jet [34-36], the coupling between heat transfer and near-wall turbulence in an impinging synthetic jet is more complicated. A synthetic jet flow induces both strong fluid temperature and velocity fluctuations near the heat transfer surface [37], due to more vigorous mixing and entrainment compared to steady jets. McGuinn et al. [37] showed a strong coherence between fluid temperature and Nusselt number at low Reynolds number. These two effects (near-wall velocity and fluid temperature fluctuations) both contribute to the resulting Nusselt number profile, which further emphasizes the value of high dynamic range velocity and turbulence measurements to study these close-coupled convection flows.

\section{Acknowledgements}

This work was supported by the Irish Research Council. The author kindly acknowledges the fruitful discussions with Darina Murray, Alan McGuinn, Rayhaan Farrelly, David Donoghue, and Ian O'Gorman.

\section{References}

1. R.D. Keane, R.J. Adrian, Meas. Sci. Technol. 1, 1202-1215 (1990)

2. M. Raffel, C. Willert, J. Kompenhans, Particle image velocimetry: A practical guide (R.J. Adrian, et al. (Eds.)) Springer: Berlin, pp. 134-146 (1998)

3. J. Westerweel, Meas. Sci. Technol. 8, 1379-1392 (1997)

4. F. Scarano, M.L. Riethmuller, Exp. Fluids 29, S51-S60 (2000)

5. M. Stanislas, K. Okamoto, C.J. Kähler, J. Westerweel, Exp. Fluids 39, 170-191 (2005)

6. A. Fincham, G. Delerce, Exp. Fluids 29, S13-S22 (2000)

7. F. Pereira, A. Ciarravano, G.P. Romano, F. Di Felice, Proc. Int. Symp. Appl. Laser Techn. Fluid Mech., Lisbon (2004)

8. R. Hain, C.J. Kähler, Exp. Fluids 42, 575-587 (2007)
9. T. Persoons, T.S. O’Donovan, Sensors 11, 1-18 (2011)

10. A. Glezer, M. Amitay, Annu. Rev. Fluid Mech. 34, 503-529 (2002)

11. J.M. Shuster, D.R. Smith, Phys. Fluids 19, 045109 (2007)

12. P. Valiorgue, T. Persoons, A. McGuinn, D.B. Murray, Exp. Therm. Fluid Sci. 33, 597-603 (2009)

13. T. Persoons, A. McGuinn, D.B. Murray, Int. J. Heat Mass Transf. 54, 3900-3908 (2011)

14. A. McGuinn, R. Farrelly, T. Persoons, D.B. Murray, Exp. Therm. Fluid Sci. 47, 241-251 (2013)

15. T. Persoons, T.S. O'Donovan, D.B. Murray, Proc. ASME Heat Transfer Summer Conf., San Francisco, CA, Vol. 1, HT2009-88440, pp. 955-962 (2009)

16. B.L. Smith, A. Glezer, AIAA J. 43, 2117-2124 (2005)

17. M.B. Gillespie, W.Z. Black, C. Rinehart, A. Glezer, J. Heat Transf.-Trans. ASME 128, 990-1000 (2006)

18. A. Pavlova, M. Amitay, J. Heat Transf.-Trans. ASME 128, 897-907 (2006)

19. Z. Travnicek, V. Tesar, Int. J. Heat Mass Transf. 46, 32913297 (2003)

20. M. Chaudhari, B. Puranik, A. Agrawal, Exp. Therm. Fluid Sci. 34, 246-256 (2010)

21. V.T. Morgan, Adv. Heat Transf. 11, 199-264 (1975)

22. G.F. Marsters, Int. J. Heat Mass Transf. 15, $921-933$ (1972)

23. L. Pera, B. Gebhart, Int. J. Heat Mass Transf. 15, 177-180 (1972)

24. K. Kitamura, F. Kami-iwa, T. Misumi, Int. J. Heat Mass Transf. 42, 4093-4106 (1999)

25. B. Gebhart, L. Pera, A.W. Schorr, Int. J. Heat Mass Transf. 13, 161-171 (1970)

26. A.W. Schorr, B. Gebhart, Int. J. Heat Mass Transf. 13, 557$571(1970)$

27. K. Noto, J. Thermophys. 3, 428-434 (1989)

28. G. Desrayaud, G. Lauriat, J. Fluid Mech. 252, 617-646 (1993)

29. E.R.G. Eckert, E.E. Soehngen, Studies on heat transfer in laminar free convection with the Zehnder-Mach interferometer, US AF Technical report 5747 (1948)

30. I.M. O'Gorman, D.B. Murray, G. Byrne, T. Persoons, Proc. ASME Int. Mech. Eng. Congr., Florida, IMECE2009-11213 (2009)

31. O. Reymond, D.B. Murray, T.S. O'Donovan, Exp. Therm. Fluid Sci. 32, 1702-1709 (2008)

32. T. Persoons, I.M. O'Gorman, D.B. Donoghue, G. Byrne, D.B. Murray, Int. J. Heat Mass Transf. 54, 5163-5172 (2011)

33. B.H. Timmins, B.L. Smith, P.P. Vlachos, Proc. ASME Fluids Eng. Div. Summer Meeting, 30724 (2010)

34. K. Kataoka, R. Sahara, H. Ase, T. Harada, J. Chem. Eng. Jpn. 20, 71-76 (1987)

35. C.J. Hoogendoorn, Int. J. Heat Mass Transf. 20, 1333-1338 (1977)

36. T.S. O'Donovan, D.B. Murray, Int. J. Heat Mass Transf. 50, 3291-3301 (2007)

37. A. McGuinn, T. Persoons, T.S. O'Donovan, D.B. Murray, Turbulence, Heat and Mass Transfer, Vol. 6 (K. Hanjalić, Y. Yagano, S. Jakirlić, Eds.), pp. 927-930 (2009)

38. M.S. Sadeghipour, M. Asheghi, Int. J. Heat Mass Transf. 37, 103-109 (1994)

39. E. Sparrow, J.E. Niethammer, J. Heat Transf.-Trans. ASME 103, 638-644 (1981)

40. M.M. Zdravkovich, Flow around circular cylinders. 1: Fundamentals, Oxford University Press (1997) 